\title{
Primary biliary cirrhosis associated with myasthenia gravis after postpartum: a case report
}

Lulu Zhang ${ }^{1}$, Dongxue Ding ${ }^{1}$, Liqiang Yu ${ }^{2}$, Huan $\mathrm{Qi}^{2}$, Chunru Han ${ }^{1}$, Jianhua Jiang ${ }^{1 *}$ and Juean Jiang ${ }^{2^{*}}$

\begin{abstract}
Background: Autoimmune diseases refers to a class of diseases involving abnormal immune response of human body and tissue damage caused by the dysregulation of autoimmune balance or destruction of immune tolerance. Recent research has revealed that the occurrence of autoimmune diseases is influenced by genetic, hormonal, immunological, and environmental factors. As sex hormone levels change obviously during pregnancy and postpartum, the morbidity and recurrence rate of autoimmune diseases increase during this period.

Case presentation: A 31-year-old Asian woman was admitted to our hospital for myasthenia gravis and treated with methylprednisolone and pyridostigmine bromide 3 months postpartum. Physical examination and laboratory inspection after admission suggested that the patient had primary biliary cirrhosis. Subsequently, azathioprine was added to the treatment, and the symptoms of both diseases were successfully controlled.

Conclusions: This case exhibits a rare condition of myasthenia gravis combined with primary biliary cirrhosis postpartum. Given the fluctuation of the immune status during the postpartum period, combined autoimmune diseases need to be taken into account when patients develop clinical symptoms of an autoimmune disease. Therefore, detailed physical and laboratory examination can help to prevent the missed diagnosis of these diseases.
\end{abstract}

Keywords: Myasthenia gravis, Primary biliary cirrhosis, Postpartum

\section{Background}

Myasthenia gravis (MG) is an acquired autoimmune disease mainly mediated by acetylcholine receptor antibody and primarily involving the acetylcholine receptor of the neuromuscular junction postsynaptic membrane. The specific hormone status during pregnancy and postpartum periods has a great effect on autoimmune diseases, potentially causing aggravation or recurrence of autoimmune diseases [1]. Studies suggest that the risk of developing MG is significantly higher in postpartum women

\footnotetext{
*Correspondence: corajiang111@126.com; jiangjuean@163.com

1 Department of Neurology, First Affiliated Hospital of Soochow

University, Suzhou 215031, China

${ }^{2}$ Department of General Medicine, First Affiliated Hospital of Soochow

University, Suzhou 215031, China
}

than in non-postpartum women [2]. Moreover, the clinical manifestations of MG are more severe in women with pregnancy or postpartum [3].

Primary biliary cirrhosis $(\mathrm{PBC})$ is a chronic intrahepatic cholestatic hepatopathy disease usually accompanied with clinical symptoms such as fatigue and itchy skin, and most commonly seen in middle-aged and elderly women. Progressive, nonsuppurative, destructive intrahepatic cholangitis is its pathologic feature and eventually leads to cirrhosis. The pathogenesis of $\mathrm{PBC}$ is not entirely understood, but it may be associated with abnormal autoimmunity caused by genetic background, environmental factors, and infection. Studies have shown that changes in sex hormones in women over time can increase the risk of $\mathrm{PBC}$ or worsen symptoms [4]. original author(s) and the source, provide a link to the Creative Commons licence, and indicate if changes were made. The images or other third party material in this article are included in the article's Creative Commons licence, unless indicated otherwise in a credit line to the material. If material is not included in the article's Creative Commons licence and your intended use is not permitted by statutory regulation or exceeds the permitted use, you will need to obtain permission directly from the copyright holder. To view a copy of this licence, visit http://creativecommons.org/licenses/by/4.0/. The Creative Commons Public Domain Dedication waiver (http://creativeco mmons.org/publicdomain/zero/1.0/) applies to the data made available in this article, unless otherwise stated in a credit line to the data. 
MG and $\mathrm{PBC}$ are two distinct autoimmune disorders, and it is rare for a patient to have both MG and PBC. Here we report a female patient who developed typical symptoms of MG after delivery, such as limb weakness that improved after rest, and with atypical symptoms of $\mathrm{PBC}$. Upon review of the literature, the patient was diagnosed as having MG with PBC.

\section{Case presentation}

A 31-year-old Asian woman presented with slurred speech and limb weakness. She first noticed slurred speech in September and subsequently developed drooping of the bilateral eyelid and, eventually, limb weakness. The above symptoms increased during periods of activity and improved after periods of rest. She was treated with pyridostigmine bromide (60 $\mathrm{mg} / 8$ hours) in a local hospital initially. The symptoms improved after taking medicine, but occasional weakness of both legs and chest tightness remained. Regarding past medical history, this patient underwent a cesarean section to give birth to her second child 3 months ago.

General physical examination revealed a yellow discoloration of facial skin and sclera. Neurological examination revealed mild dysarthria, mild droop of both side ptosis, and generalized muscle weakness after exercise. Weakness was most pronounced in the lower extremities. The upper eyelid fatigue test score was 2 . Repetitive nerve stimulation showed that the amplitude of right axillary nerve decreased significantly at 1,3 , and $5 \mathrm{~Hz}$, with a amplitude reduction of $35.6 \%, 45.2 \%$, and $62.8 \%$ respectively. Besides, the amplitude of left facial nerve decreased by $13.9 \%$ and $17.5 \%$ at $3 \mathrm{~Hz}$ and $5 \mathrm{~Hz}$. Reduced density of liver parenchyma is observed on contrastenhanced chest computerized tomography (CT), and fatty liver may have been present. Positive results of laboratory tests are presented in Table 1. Rheumatoid factors, anti-nuclear antibody, anti-smooth muscle antibody and anti-thyroid antibody were negative. Thyroid hormone levels were within normal intervals. According to the clinical, electromyographic, and laboratory findings, the diagnosis of MG was unambiguous. In addition, the patient's liver enzymes were elevated with positive AMAM2 serology; according to the guidelines [5], she was diagnosed with PBC.

The patient was diagnosed with MG after she came to our hospital. Therefore, she was treated with pyridostigmine bromide (60 mg/8 hours) and methylprednisolone after admission. The initial dose of methylprednisolone was $30 \mathrm{mg} /$ day, then gradually increased to $80 \mathrm{mg} /$ day for maintenance 3 days, and finally decreased to $30 \mathrm{mg} /$ day for maintenance treatment. Ursodeoxycholic acid (250 mg/8 hours) and azathioprine (50 mg/12 hours) treatment was added after diagnosis of PBC. The patient's
Table 1 Positive results of laboratory tests

\begin{tabular}{lll}
\hline Item & Result & Normal range \\
\hline ESR $(\mathrm{mm} / \mathrm{hour})$ & 34.00 & $0-26$ \\
AchR-IgG $(\mathrm{U} / \mathrm{mL})$ & 12.10 & $<0.45$ \\
TBIL $(\mu \mathrm{mol} / \mathrm{L})$ & 44 & $3.4-17.1$ \\
$\mathrm{DBIL}(\mu \mathrm{mol} / \mathrm{L})$ & 14 & $0-6.8$ \\
$\mathrm{IBIL}(\mu \mathrm{mol} / \mathrm{L})$ & 30 & $1.7-10.2$ \\
$\mathrm{ALT}(\mathrm{U} / \mathrm{L})$ & 115.4 & $7-40$ \\
AST $(\mathrm{U} / \mathrm{L})$ & 68.8 & $13-35$ \\
AMA-M2 & Positive $(++)$ & Negative \\
\hline
\end{tabular}

ESR erythrocyte sedimentation rate, $A c h R$-IgG anti-acetylcholine receptor immunoglobulin G, TBIL total bilirubin, DBIL direct bilirubin, IBIL indirect bilirubin, ALT alanine transaminase, AST aspartate transferase, AMA-M2 antimitochondrial antibody $\mathrm{M} 2$

condition improved steadily during 1 month of hospitalization. She continued to receive a combined treatment with pyridostigmine bromide $(60 \mathrm{mg} / 8$ hours $)$, methylprednisolone (16 mg/12 hours), ursodeoxycholic acid ( $250 \mathrm{mg} / 8$ hours), and azathioprine $(50 \mathrm{mg} / 12$ hours) after discharge.

During the 2 years follow-up after discharge, the patient's methylprednisolone was gradually reduced to discontinuation within 1 year. Daily ursodeoxycholic therapy was administered strictly according to our instructions for 6 months after discharge, and the patient ceased ursodeoxycholic on her own. Interestingly, her liver enzyme indexes were all normal, although she stopped taking ursodeoxycholic acid after discharge. Now, the patient is receiving long-term maintenance treatment with pyridostigmine bromide $(60 \mathrm{mg} / 8$ hours) and azathioprine $(50 \mathrm{mg} /$ day). During the 2 years followup, the patient had no obvious discomfort except weight gain, and the relevant liver enzyme indexes were normal.

\section{Discussion and conclusions}

This report firstly described the occurrence of MG and $\mathrm{PBC}$ in a patient in the postpartum period. In previous reports, the use of $\mathrm{D}$-penicillamine in patients with $\mathrm{PBC}$ resulted in the development of MG [6]. However, patients can also develop both PBC and MG without D-penicillamine $[7,8]$. In addition, it has been reported that patients could develop other autoimmune diseases (AID) in addition to PBC and MG [9]. According to previous reports, $\mathrm{PBC}$ combined with MG tends to occur more frequently in middle-aged and elderly women than men of the same age. This suggested that women can be more susceptible to autoimmune diseases in some conditions.

Studies have suggested that more than $80 \%$ of AID occur in women of childbearing age [10,11], and AID is prone to relapse or aggravation during pregnancy and postpartum and improves after menopause [12]. 
The above phenomenon further confirmed that hormonal changes play an essential role in the occurrence and development of AID. The risk of AID is significantly increased in the first postpartum year and then decreased gradually due to the changes of sex hormones during pregnancy and postpartum [13, 14]. Serum levels of estradiol, progesterone, prolactin, and cortisol increase gradually during pregnancy. Estrogen and progesterone drop suddenly after childbirth, but prolactin levels remain high, especially in breastfeeding mothers [1]. Moreover, breastfeeding further reduces estrogen and cortisol levels $[15,16]$. Changes in hormone levels lead to a strong immune tolerance during pregnancy, while the immune environment shifts to a proinflammatory state at the end of pregnancy and postpartum. The volatility of the immune state leads to bodies' susceptibility to AID during this period.

This report plays a reference role in the diagnosis and treatment of AID in postpartum patients. Given the changes in immune status, it should be determined whether some other autoimmune diseases were complicated in the postpartum patients diagnosed with AID. Careful physical and laboratory examination can help to prevent the missed diagnosis of these diseases.

\section{Abbreviations}

AID: Autoimmune diseases; MG: Myasthenia gravis; PBC: Primary biliary cirrhosis; CT: Computed tomography; AMA: Anti-mitochondrial antibodies; ESR: Erythrocyte sedimentation rate; AchR-IgG: Anti-acetylcholine receptor immunoglobulin G; TBIL: Total bilirubin; DBIL: Direct bilirubin; IBIL: Indirect bilirubin; ALT: Alanine transaminase; AST: Aspartate transferase; AMA-M2: Antimitochondrial antibody $\mathrm{M} 2$.

\section{Acknowledgements}

Not applicable.

\section{Authors' contributions}

LLZ and DXD contributed equally to this work. LLZ and DXD participated in the preliminary draft and medical data collection. LQY and HQ were involved in treatment planning. $\mathrm{CRH}$ participated in the follow-up of patients after discharge. JHJ and JAJ contributed to the interpretation of the findings and critical revision of the manuscript. All authors read and approved the final manuscript.

\section{Funding}

Not applicable.

\section{Availability of data and materials}

The datasets used and/or analyzed during the current study are available from the corresponding author on reasonable request

\section{Declarations}

Ethics approval and consent to participate Not applicable.

\section{Consent for publication}

Written informed consent was obtained from the patient for publication of this case report and any accompanying images. A copy of the written consent is available for review by the Editor-in-Chief of this journal.

\section{Competing interests}

The authors declare that they have no competing interests.

Received: 22 July 2021 Accepted: 2 September 2021

Published online: 10 October 2021

\section{References}

1. Borba W, Zandman-Goddard G, Shoenfeld Y. Exacerbations of autoimmune diseases during pregnancy and postpartum. Best Pract Res Clin Endocrinol Metab. 2019:33(6):101321.

2. Boldingh Ml, Maniaol AH, Brunborg C, Weedon-Fekjaer H, Verschuuren JJ, Tallaksen CM. Increased risk for clinical onset of myasthenia gravis during the postpartum period. Neurology. 2016;87(20):2139-45.

3. Djelmis J, Sostarko M, Mayer D, Ivanisevic M. Myasthenia gravis in pregnancy: report on 69 cases. Eur J Obstet Gynecol Reprod Biol. 2002;104(1):21-5.

4. Sun Y, Haapanen K, Li B, Zhang W, Van de Water J, Gershwin ME. Women and primary biliary cirrhosis. Clin Rev Allergy Immunol. 2015;48(2-3):285-300.

5. Lindor KD, Bowlus CL, Boyer J, Levy C, Mayo M. Primary biliary cholangitis: 2018 practice guidance from the American Association for the Study of Liver Diseases. Hepatology. 2019;69(1):394-419.

6. Marcus SN, Chadwick D, Walker RJ. D-penicillamine-induced myasthenia gravis in primary biliary cirrhosis. Gastroenterology. 1984;86(1):166-8.

7. Horigome H, Nomura T, Saso K, Joh T, Ohara H, Akita S, Sobue S, Mizuno $Y$, Kato Y, Itoh M. Coexistence of primary biliary cirrhosis and myasthenia gravis: a case study. Hepatogastroenterology. 2000:47(31):125-7.

8. Finsterer J, Hoflich S. Successful low-dose azathioprine for myasthenia gravis despite hepatopathy from primary sclerosing cholangitis: a case report. J Med Case Rep. 2010;4:356.

9. Rajaraman S, Deodhar SD, Carey WD, Salanga VD. Hashimoto's thyroiditis, primary biliary cirrhosis, and myasthenia gravis. Am J Clin Pathol. 1980;74(6):831-4.

10. Nussinovitch U, Shoenfeld $Y$. The role of gender and organ specific autoimmunity. Autoimmun Rev. 2012;11(6-7):A377-385.

11. Shoenfeld Y, Zandman-Goddard G, Stojanovich L, Cutolo M, Amital H, Levy Y, Abu-Shakra M, Barzilai O, Berkun Y, Blank M, et al. The mosaic of autoimmunity: hormonal and environmental factors involved in autoimmune diseases-2008. Isr Med Assoc J. 2008;10(1):8-12.

12. Zandman-Goddard G, Peeva E, Shoenfeld Y. Gender and autoimmunity. Autoimmun Rev. 2007;6(6):366-72.

13. Khashan AS, Kenny LC, Laursen TM, Mahmood U, Mortensen PB, Henriksen TB, O'Donoghue K. Pregnancy and the risk of autoimmune disease. PLoS ONE. 2011;6(5):e19658.

14. Ortona E, Pierdominici M, Maselli A, Veroni C, Aloisi F, Shoenfeld Y. Sex-based differences in autoimmune diseases. Ann Ist Super Sanita. 2016;52(2):205-12.

15. Brennan P, Ollier B, Worthington J, Hajeer A, Silman A. Are both genetic and reproductive associations with rheumatoid arthritis linked to prolactin? Lancet. 1996;348(9020):106-9.

16. Thayer ZM, Agustin Bechayda S, Kuzawa CW. Circadian cortisol dynamics across reproductive stages and in relation to breastfeeding in the Philippines. Am J Hum Biol. 2018;30(4):e23115.

\section{Publisher's Note}

Springer Nature remains neutral with regard to jurisdictional claims in published maps and institutional affiliations. 